\title{
IsSUE BRIEF
}

\section{Optimization of Interventions in Ebola: Differential Contagion}

Amesh A. Adalja and D. A. Henderson

M ANAGING ANY CONTAgIOUS INFECTIOUS DISEASE outbreak involves breaking the chain of transmission from those who are infected with the pathogen to those who are not. Not all pathogens, however, are equal in their contagiousness, and considerable variation exists.

The viral disease measles, for example, is considered to be one of the most contagious human diseases. Its high rate of contagion is driven by 2 attributes: the ability to spread through the air via small particles (ie, airborne transmission) and the fact that one of the symptoms of measles is coughing, an effective means of expelling those particles. On average, a person infected with measles can infect 15 other people through the course of his or her illness. ${ }^{1}$ Diseases like tetanus and anthrax, on the other hand, are not contagious at all because they lack the ability to spread between humans. In between these 2 extremes lie all the other infectious diseases.

Another factor that affects infectiousness is the course of illness. Disease symptoms such as coughing, vomiting, and diarrhea can serve to heighten the transmission of a pathogen. For example, a person with whooping cough is more contagious when he is coughing than when he is not because the expelling of infectious material renders him more contagious.

Ebola viral disease (EVD) is no different. Ebola is spread exclusively through blood and bodily fluids. Thus, those Ebola patients experiencing symptoms of vomiting and diarrhea-because they are literally expelling infectious bodily fluids - would be expected to be more contagious than those without these symptoms, who may have less opportunity to spread the illness.

Another feature of Ebola is the hemorrhagic manifestations that occur in approximately half of patients. This often terminal manifestation includes bleeding from multiple bodily orifices, IV sites, needle punctures, and gums. An individual who is hemorrhaging, much like the vomiting patient, would be expected to be more contagious since viral particles are being emitted into the environment from the patient's body via blood.

The phenomenon of varied contagiousness was seen in the 1972 smallpox outbreak in the former Yugoslavia, in which 1 individual with hemorrhagic smallpox spread the virus to 38 contacts. Other victims transmitted infection to only 2 to 3 others. $^{2}$

If this differential contagiousness occurs with Ebola as well, it could be employed to optimize isolation and contact tracing activities_-essential in a resource-challenged setting such as West Africa. If those individuals with hemorrhagic disease, vomiting, and diarrhea are the most infectious, then infection control should be prioritized to ensure that these patients are cared for with the appropriate protective measures in place. When contact tracing activities are performed, prioritizing the location of those who had contact with such cases could occur. Close contacts should be identified whenever possible and either kept under surveillance throughout 1 incubation period or provided preventive vaccination or treatment, as appropriate when available.

Amesh A. Adalja, MD, is a Senior Associate, and D. A. Henderson, MD, MPH, is Distinguished Scholar, both at the UPMC Center for Health Security, Baltimore, Maryland. 
Additionally, each case of Ebola identified should prompt a concurrent epidemiologic investigation to identify the source of the particular infection. Such actions ensure that primary cases are still being identified and also assess the efficacy of control measures such as isolation.

The approach outlined serves to focus response efforts on activities anticipated to have the highest impact on slowing disease transmission by allocating scarce epidemiologic and infection control resources optimally.

\section{REFERENCES}

1. Gay NJ. The theory of measles elimination: implications for the design of elimination strategies. J Infect Dis 2004;189: S27-S35.

2. Public Health Service. Smallpox in Yugoslavia. September 22, 1972. http://www.nlm.nih.gov/nichsr/esmallpox/Smallpox Yugoslavia-EPI-72-91-2.pdf. Accessed August 20, 2014. 